This paper is the preprint version of the paper "Classification of URL bitstreams using bag of bytes" published by IEEE with DOI: 10.1109/ICIN.2018.8401597.

(C) 2021 IEEE. Personal use of this material is permitted. Permission from IEEE must be obtained for all other uses, in any current or future media, including reprinting/republishing this material for advertising or promotional purposes, creating new collective works, for resale or redistribution to servers or lists, or reuse of any copyrighted component of this work in other works. 


\title{
Classification of URL bitstreams using Bag of Bytes
}

\author{
Keiichi Shima*, Daisuke Miyamoto ${ }^{\dagger}$, Hiroshi Abe*, Tomohiro Ishihara ${ }^{\ddagger}$, \\ Kazuya Okada ${ }^{\ddagger}$, Yuji Sekiya ${ }^{\ddagger}$, Hirochika Asai ${ }^{\S}$ and Yusuke Doi ${ }^{\S}$ \\ * Internet Initiative Japan Inc., 2-10-1 Fujimi, Chiyoda-ku, Tokyo 102-0071, Japan \\ Email: keiichi@iijlab.net and abe@iij.ad.jp \\ $\dagger$ Nara Advanced Institute of Science and Technology, 8916-5 Takayama-cho, Ikoma, Nara 630-0192, Japan \\ Email: daisu-m@is.naist.jp \\ $\ddagger$ The University of Tokyo, 7-3-1 Hongo, Bunkyo-ku, Tokyo 113-8654, Japan \\ Email: sho@c.u-tokyo.ac.jp, okada@ecc.u-tokyo.ac.jp and sekiya@nc.u-tokyo.ac.jp \\ $\S$ Preferred Networks, Inc., 1-6-1 Otemachi, Chiyoda-ku, Tokyo 100-0004, Japan \\ Email: asai@preferred.jp and doi@preferred.jp
}

\begin{abstract}
Protecting users from accessing malicious web sites is one of the important management tasks for network operators. There are many open-source and commercial products to control web sites users can access. The most traditional approach is blacklist-based filtering. This mechanism is simple but not scalable, though there are some enhanced approaches utilizing fuzzy matching technologies. Other approaches try to use machine learning (ML) techniques by extracting features from URL strings. This approach can cover a wider area of Internet web sites, but finding good features requires deep knowledge of trends of web site design. Recently, another approach using deep learning (DL) has appeared. The DL approach will help to extract features automatically by investigating a lot of existing sample data. Using this technique, we can build a flexible filtering decision module by keep teaching the neural network module about recent trends, without any specific expert knowledge of the URL domain. In this paper, we apply a mechanical approach to generate feature vectors from URL strings. We implemented our approach and tested with realistic URL access history data taken from a research organization and data from the famous archive site of phishing site information, PhishTank.com. Our approach achieved $2 \sim 3 \%$ better accuracy compared to the existing DLbased approach.
\end{abstract}

\section{INTRODUCTION}

As the Internet grows and becomes more stable, the importance of its networking function as a social infrastructure becomes greater and greater. Although abuse was observed from the beginning of the Internet, as the Internet is being relied on by business activities etc., business-oriented attacks have increased. From the viewpoint of network management, we need to consider several different kinds of threats to protect both network operational stability and the users who are connected and using the network. We need to protect against (D)DoS attacks, unintended network accesses, virus infection, and so on.

Phishing is one such threat to which network operators must pay attention. An attacker tries to make users access faked sites that look similar to existing web service pages, typically banking sites or shopping sites. In such faked pages, the attacker provides a faked login screen to steal account numbers and passwords of victims. A document[1] published by Anti-Phishing Working Group ${ }^{1}$ revealed that more than 1.2 million phishing attacks were reported in 4Q 2016 which is $65 \%$ larger than 4Q 2015. Because the document covered only reported attacks, we can guess there was more hidden or unnoticed attacks in reality.

Network operators need to protect their users from accessing such malicious sites. If you are an operator of an ISP or a similar kind of service aggregator, then you need to take care of your customer's network service operations too. Since the number of phishing sites is huge and growing (100 of thousands of unique sites are reported in the document[1] and they keep changing their site locations), we need an automated and adaptive approach to defend customers from such activities.

The rest of the paper is structured as follows. We discuss related work in Section II. Our proposed idea to form a unique URL feature vector is introduced in Section III. The neural network topology used to classify URLs in our experiments is disclosed in Section IV. The datasets and evaluation results are explained in Section $\mathrm{V}$ and $\mathrm{VI}$. We conclude the achievementss and future directions of this work in SectionVII

\section{RELATED WORK}

Garera et al. proposed a framework to detect phishing sites using machine learning technique in [2]. They defined several features which they thought important to classify benign URLs and malicious URLs. Examples of the features are a page rank value, a type of domain names (e.g. whether the host part consists of IP address numbers or a hostname, whether the number of sub-domains are too large, etc), and so on. Ma et al. proposed [3] a URL classification method using very high dimensional feature vectors generated from lexical features of URL strings and host name features such as reputation. Prakash et al. proposed a new blacklist generation mechanism in [4]. Their idea is that attackers often use similar URLs to those they were using before and use slightly modified strings in new phishing attacks. They proposed some rules to generate potential blacklist URLs to protect future phishing attacks.

\footnotetext{
${ }^{1}$ https://antiphishing.org/
} 


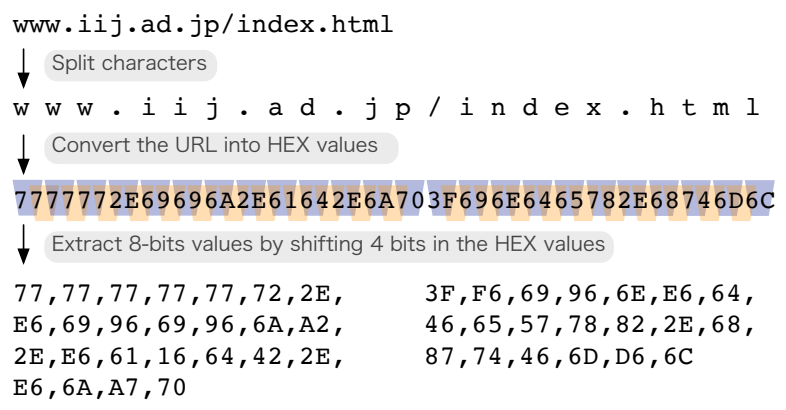

Fig. 1. The procedure to extract byte values from a URL string

So far, the previous work is based on string analysis and external information (such as page rank) to define feature vectors of URLs. Since they are based on expert knowledge studied when they were working on the classification issue, if the assumptions of the knowledge changes then the mechanism may lose accuracy.

The other approach is to inspect the contents of the target URLs. Zhang et al. proposed a phishing site detection mechanism by looking into the contents of the target sites [5]. This approach apparently works more precisely; however accessing the target sites causes different issues, such as network traffic overhead, possible risks to access (possibly) malicious contents.

Saxe et al. proposed a deep neural networking-based approach named eXpose[6] to classify URLs, file names, and registry keys. In contrast to the past works, they tried not to define any feature vectors using expert knowledge. Instead, they tried to offload the work to extract feature vectors to their deep neural network model. They expand each character of a URL string using the embedding technique and apply four different sizes of convolutional neural networking layers to extract URL features. In their paper, their approach achieved better accuracy than an expert knowledge based feature extraction approach and an $\mathrm{N}$-gram based feature extraction approach. They imply better accuracy may be achieved by using different URL vectorization methods and/or different neural network topologies. In this paper, we follow this approach and try to achieve simpler and better result.

\section{BAG OF BYTES AND URL VECTOR}

We tried not to use any expert knowledge of a specific domain, in this case, knowledge of URL structures. In that sense, the direction of our approach is same as eXpose[6] took. In eXpose, they used the last 200 characters of a URL string and converted it into a $200 \times 32$-dimensional vector using an embedding technique. The reason why they cut the string to 200 characters is that $95 \%$ of URL strings are shorter than 200 characters in their investigation. In our approach, we also try to convert URL strings mechanically but use all the characters included in the URL strings.

Fig. 1 shows how a URL string is converted. The basic idea is to count byte values included in the URL string. The point is that we extract byte values not only from each character but also overlapping parts of neighboring characters by shifting 4-bits when extracting byte values. By using

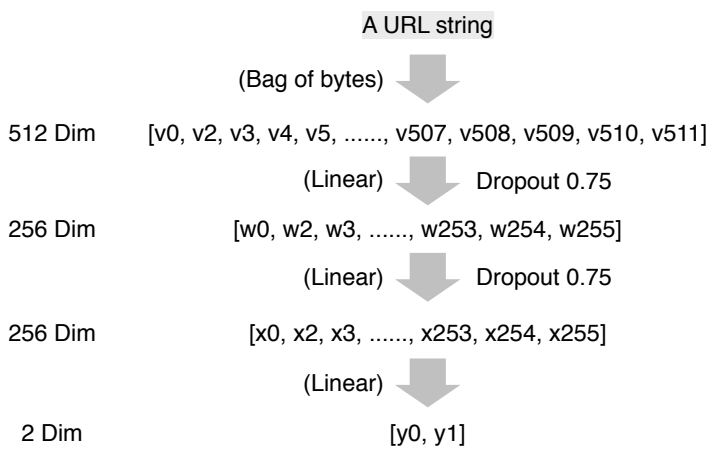

Fig. 2. The neural network model for URL classification

intersecting bits between two neighboring cahracters, we can embed combination information of two characters appearing sequentially. After extracting the byte values, we count how many times each value appears in the original URL string. We perform the extraction operation for both the host part and the path part separately, and achieve a 512-dimensional vector that represents the original URL. Finally, each vector is normalized and we treat the final vector value as a URL vector.

\section{NeURAL Network TOPOLOGY}

The neural network topology we used for classification is not complex. The topology is a basically multi-layer linear (or sometimes called a 'dense') topology. Fig. 2 depicts the neural network topology for URL classification.

The first layer is a 512-dimensional array of nodes which receives the vector value of a URL, the first half comes from the host part, the latter part comes from the path part. The value will be passed to a 256-dimension array twice using linear connections. To suppress over-fitting, links are dropped out with the ratio of 0.75 . The dropout ratio value is decided based on our experience of several trials of training. Finally, the 256-dimension array will be mapped to two classes that indicate whether the input URL is a benign URL or a malicious URL.

\section{DATASETS}

The datasets we used to evaluate the proposed model consist of two sources. One is a set of URLs retrieved from the PhishTank.com ${ }^{2}$ The site provides information on phishing sites based on the reports submitted by users. The submitted URLs are manually checked to see if the URL is a real phishing site; if so, it is marked as active. PhishTank.com distributes latest active phishing site list which can be used for access filtering. We retrieved the recorded set of phishing URLs and used them as a blacklist.

For a whitelist, we collected access log data of a research organization. A difficult point is that it is impossible to prepare a pure white URL access list from a real access log. There is always the possibility that the list we collected includes malicious URLs. To minimize the effect, we try to clean up the access list by removing all the URL entries listed in the phishing site list retrieved from PhishTank.com.

\footnotetext{
${ }^{2}$ https://www.phishtank.com/
} 
TABLE I. URL DATASETS FOR TRAINING

\begin{tabular}{|c|c|c|}
\hline Type & Content & Count \\
\hline Blacklist 1 & $\begin{array}{l}\text { Phishing site URLs reported at PhishTank.com before } \\
2017-04-25 \text {. This list is used as a blacklist for learning } \\
\text { and testing in conjunction with the Whitelist } 1 \text {. }\end{array}$ & 26,722 \\
\hline Blacklist 2 & $\begin{array}{l}\text { Phishing site URLs reported at PhishTank.com before } \\
2017-10-03 \text {. This list is used to cleanse the target } \\
\text { access } \log \text { captured at the anonymous research or- } \\
\text { ganization X. }\end{array}$ & 68,172 \\
\hline Whitelist 1 & $\begin{array}{l}\text { A sampled list of URL access log captured at the } \\
\text { anonymous research organization X on } 2017-04-25 \\
\text { excluding the entries listed in the Blacklist } 2 \text {. This } \\
\text { list is used for learning and testing in conjunction } \\
\text { with the Blacklist } 1 .\end{array}$ & 26,722 \\
\hline
\end{tabular}

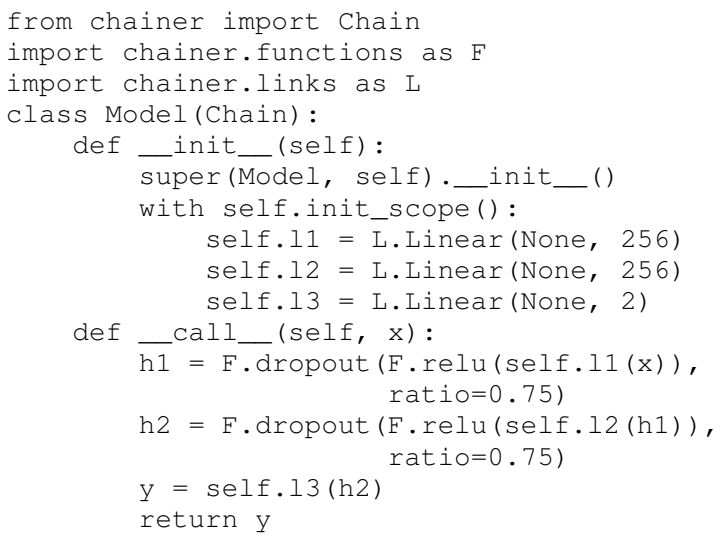

Fig. 3. The code fragment that implements our proposed neural network model using Chainer

Table II shows the datasets we prepared for training. The number of entries in each dataset is also shown in the table.

The main target is the URL access list captured at an anonymous research organization $X$ on 2017-04-25. The access list contains more than 142 million entries. The list contains not only benign URLs but also phishing site URLs. We tried to clean the list with the phishing site URL data reported at PhishTank.com from 2017-04-24 to 2017-10-03. Using the blacklist data, including future entries beyond the target data, will help to remove some of URLs that had not been found at the day of 2017-04-25 and make the white URL a bit whiter.

We prepared a balanced dataset to fit the neural network for both malicious URL features and benign URL features evenly. Since the number of the white URSs was larger than that of the black URLs, we first picked 10,000 entries of the URL access $\log$ from each hour, i.e. 240,000 entries, and randomly selected the 26,722 entries from the list which was the same size as Blacklist 1.

\section{EVAluation}

We implemented our idea described in section IV using Chainer ${ }^{3}$. The code of the model is shown in Fig. 3.

We used the datasets shown in Table I] The URL entries included in the Blacklist 1 and the Whitelist 1 are mixed and randomly shuffled. The ratio of training and validating is $80 \%$ and $20 \%$. The mini-batch size is set to 100 , and the number of epochs is 20 .

$3^{3}$ https://chainer.org
TABLE II. RESULTS OF ACCURACY AND TRAINING TIME USING WHITELIST 1 AND BLACKLIST 1 IN TABLE[

\begin{tabular}{|c||c|r|r|}
\hline & Optimizer & Accuracy (\%) & Training time (s) \\
\hline \hline Our method & Adam & 94.18 & 32 \\
\hline- & AdaDelta & 93.54 & 31 \\
\hline- & SGD & 88.29 & 31 \\
\hline \hline eXpose[6] & Adam & 90.52 & 119 \\
\hline- & AdaDelta & 91.31 & 119 \\
\hline- & SGD & 77.99 & 116 \\
\hline
\end{tabular}

TABLE III. URL DATASETS FOR PREDICTING

\begin{tabular}{|l||l|c|}
\hline Type & Content & Count \\
\hline \hline Blacklist 3 & $\begin{array}{l}\text { Phishing site URLs reported at PhishTank.com before } \\
\text { 2017-05-25. This list is used as a black list for } \\
\text { learning and testing in conjunction with the white list. }\end{array}$ & 39,776 \\
\hline Whitelist 2 & $\begin{array}{l}\text { A sampled list of URL access log captured at the } \\
\text { anonymous research organization X on 2017-05-25 } \\
\text { excluding the entries listed in the Blacklist 2. This } \\
\text { list is used for test the neural network model trained } \\
\text { with the Blacklist 1 and Whitelist } 1 .\end{array}$ & 39,776 \\
\hline
\end{tabular}

We tested our neural network model using three different optimizers, Adam, AdaDelta, and SGD. Among them, Adam was the best optimizer with an accuracy of $94.18 \%$.

As mentioned above, eXpose [6] tried to classify URLs using a convolutional neural network. Unfortunately, while they described their neural network model, they didn't provide their code and dataset used in their evaluation. In their paper, they said they achieved more than $99.9 \%$ accuracy. To compare their approach to ours, we implemented their neural network model using Chainer and evaluated it with the same dataset we used for our cases. The results are also shown in the same table. Although the result using SGD as an optimizer was a bit low; however, with the other optimizers, their approach achieved almost same but a bit lower accuracy than ours. We also measured the time consumed for training because they are using more complex neural networking topology. Their model requires four times more training time than ours.

Fig. 4 shows the learning curves (accuracy and loss values at each epoch). eXpose quickly converged to the stable state compared to our method; although the final accuracy is lower than ours using our datasets. When looking at the loss values, eXpose looks to be over-fitting when the count of epochs increase. Our proposal uses a dropout ratio of 0.75 between neural network layers to suppress over-fitting, while eXpose uses 0.5 as is specified in the eXpose paper. The larger dropout value may contribute less over-fitting in the eXpose case.

We tried to apply the neural network model trained with the dataset of Blacklist 1 and Whitelist 1 on a different dataset containing data captured later than the training data as shown in Table III The evaluation results are shown in Table IV] Our method achieved $95.17 \%$ of accuracy with 0.9525 of Fmeasure score. We tried to predict the same dataset with the eXpose model trained with the same trainer dataset too. The results are also shown in Table IV eXpose achieved good but slightly lower score than our method. The Receiver Operating Characteristic (ROC) curves and Area Under the Curve (AUC) values are shown in Fig. 5

It is difficult to say if our neural network model is applicable to a specific real operation or not given the results 

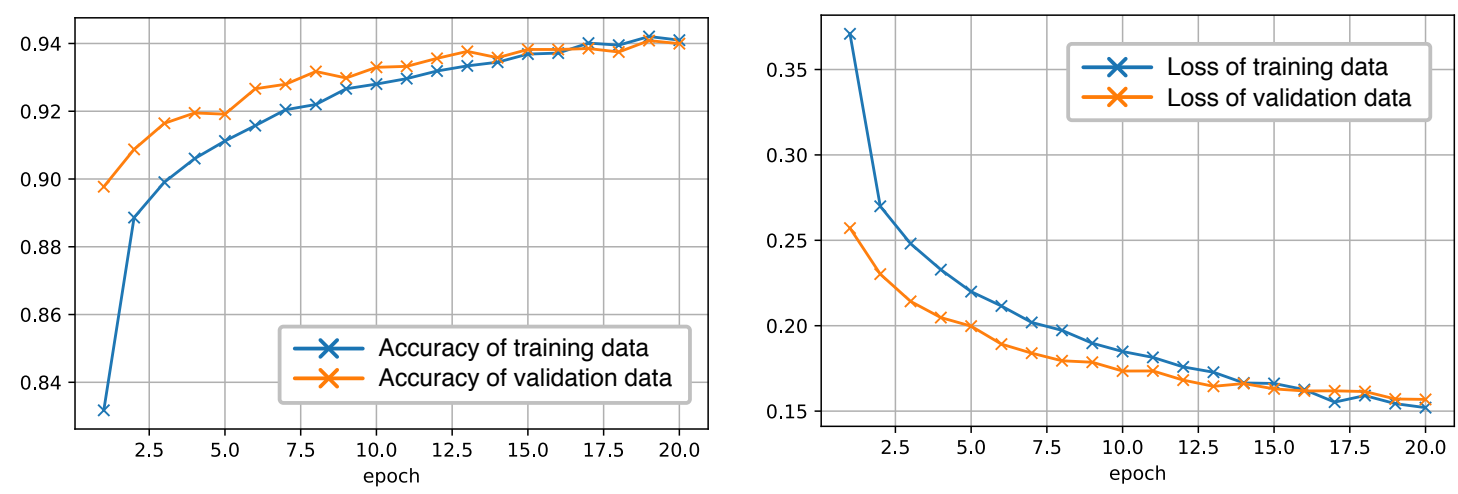

(a) Our method (optimizer $=$ Adam)
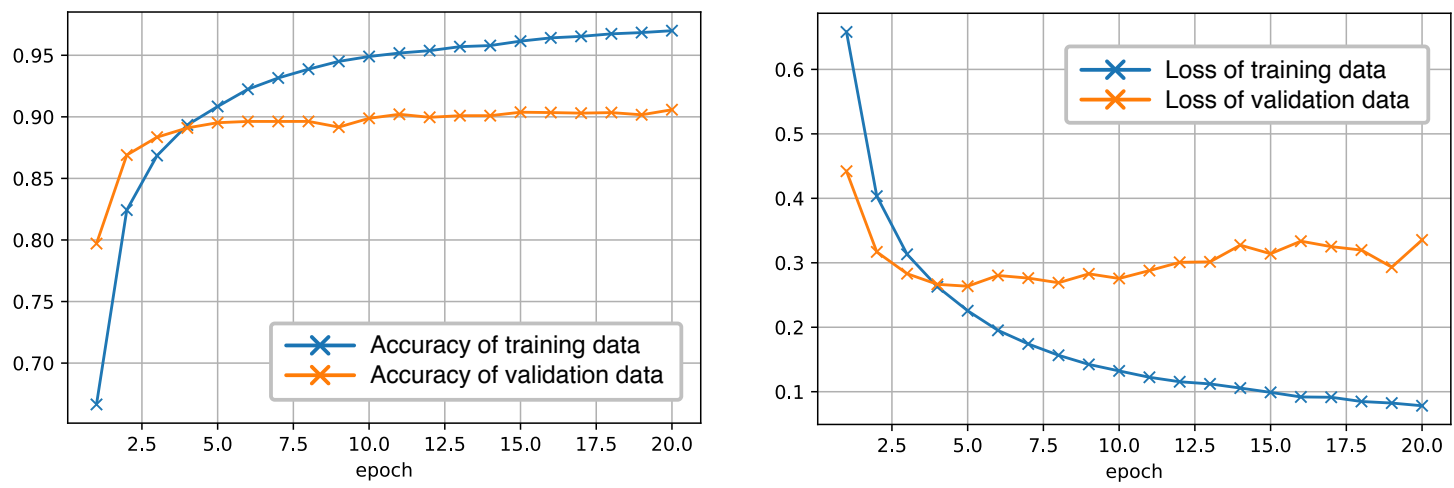

(b) eXpose (optimizer $=$ Adam)

Fig. 4. Learning curves of our method (a) and eXpose (b): The blue lines indicate results of training data and the orange lines indicate results of validation data of each epoch while training each model.

TABLE IV. PREDICTION RESULTS OF THE DATASET SHOWN IN TABLE[IIUSING THE TRAINED NEURAL NETWORK MODEL WITH THE DATASET SHOWN IN TABLE!

\begin{tabular}{|l||r|r|r|r|}
\hline & Accuracy (\%) & Precision (\%) & Recall (\%) & F-measure \\
\hline \hline Our method & $95.17 \%$ & $93.76 \%$ & $96.78 \%$ & 0.9525 \\
\hline eXpose & $92.99 \%$ & $93.00 \%$ & $92.99 \%$ & 0.9299 \\
\hline
\end{tabular}

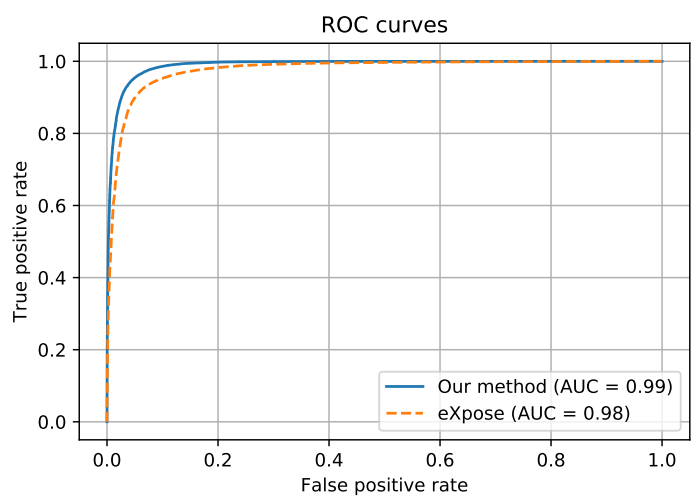

Fig. 5. ROC curves and AUC values measured with the prediction datasets as shown in Table III using our model and eXpose model

shown only in this paper. The accuracy may change depending on the target environment. The eXpose paper showed their classification accuracy was more than $99.9 \%$; however as we show, if we change the dataset the result changes. For the same reason, if the data sources are changed, our mechanism may produce lower accuracy than in this paper.

One of the issues when working on this type of research is that using a generalized dataset is really difficult. The approaches sometimes optimized to the target datasets, which are the only datasets that the authors can access in many cases. In the image recognition field, researchers have several common datasets such as MNIST ${ }^{4}$ that can be used to evaluate each researchers' proposal with a same baseline. Probably we need to start the effort to build shared datasets for network data too.

\section{CONCLUSION}

In this paper, we proposed a new neural network model for classifying URLs into benign and phishing. The learning overhead of the proposed network is light because the topology consists of just three layers of linear networks. Shorter learning time make it possible to try with many different kinds of data to optimize the neural network topology. Since the accuracy is sometimes affected by the quality of training data, more trials may result in more suitable networks.

The debatable point is that it is not possible to prove which method is better or best. In this area, analyzing network log data using neural network technologies, we lack a common

\footnotetext{
${ }^{4}$ http://yann.lecun.com/exdb/mnist/
} 
dataset to compare approaches. In this paper, we could achieve better performance than the past work, however, it might be worse if we used other datasets. Thus, all the researchers in this community need to start building a dataset to share among researchers working on network log analysis using machine/deep learning approaches to improve the technologies.

The datasets we used in this paper were taken from the famous phishing URL archive site PhishTank.com and from an anonymous research organization's network. With these realistic datasets, our model resulted in $94.18 \%$ accuracy in the training/validation phase and $95.17 \%$ when classifying newer dataset then the training phase, which was higher than the previously proposed neural network model published in [6] which uses more complex neural network topology.

\section{ACKNOWLEDGMENT}

This work was supported by JST CREST Grant Number JPMJCR1783, Japan.

\section{REFERENCES}

[1] "Phishing activity trends report - 4th quarter 2016," Anti-Phishing Working Group, Inc., Tech. Rep., February 2017.

[2] S. Garera, N. Provos, M. Chew, and A. D. Rubin, "A framework for detection and measurement of phishing attacks," in Proceedings of the 2007 ACM Workshop on Recurring Malcode, ser. WORM '07. New York, NY, USA: ACM, November 2007, pp. 1-8.

[3] J. Ma, L. K. Saul, S. Savage, and G. M. Voelker, "Beyond blacklists: Learning to detect malicious web sites from suspicious URLs," in Proceedings of the 15th ACM SIGKDD International Conference on Knowledge Discovery and Data Mining, ser. KDD '09. New York, NY, USA: ACM, June 2009, pp. 1245-1254.

[4] P. Prakash, M. Kumar, R. R. Kompella, and M. Gupta, "PhishNet: Predictive blacklisting to detect phishing attacks," in 2010 Proceedings IEEE INFOCOM, ser. INFOCOM, 2010, pp. 1-5.

[5] Y. Zhang, J. I. Hong, and L. F. Cranor, "CANTINA: A content-based approach to detecting phishing web sites," in Proceedings of the 16th international conference on World Wide Web, ser. WWW '07. ACM, May 2007, pp. 639-648.

[6] J. Saxe and K. Berlin, "eXpose: A character-level convolutional neural network with embeddings for detecting malicious URLs, file paths and registry keys," CoRR, vol. abs/1702.08568, February 2017. 